\title{
Thermal noise and the branching threshold in brittle fracture
}

\author{
L. M. Sander ${ }^{1}$ and S.V. Ghaisas ${ }^{2}$ \\ ${ }^{1}$ Physics Department, The University of Michigan, Ann Arbor MI 48105-1120. USA \\ 2 Department of Electronics Science, University of Pune, Pune 411007, India
}

(May 11, 2018)

\begin{abstract}
Many studies have confirmed that cracks in brittle materials branch when the crack speed exceeds a certain threshold, $v_{c}$, but the value of that threshold is not understood. Almost all theoretical calculations overestimate $v_{c}$ by factors of two or more. We show that thermal noise can reduce the threshold by a substantial amount, and we propose that this effect can account for the discrepancy.
\end{abstract}

The formation of cracks in the fracture of solids leads to extremely intricate pattern formation. One of the most interesting phenomena in this field is the mirror-misthackle transition in brittle fracture, for example, that of glass [1]. This occurs when a crack accelerates and passes through a critical velocity, $v_{c}$ above which it becomes unstable against branching [2-5]. Below $v_{c}$ the surface created by the crack is smooth (mirror-like) while above it is rough. However, the value of $v_{c}$ is not theoretically understood: typically, theoretical estimates are too large [6,6]. In this paper we will show that including thermal noise in the theory can lower the threshold substantially.

For many conditions, once started, cracks accelerate until their velocity is of the order of the free surface wave (Rayleigh) velocity $c_{R}$. In the case of a Mode I crack, the crack cannot move faster than the sides can move apart: that is, faster than $c_{R}$. Thus $c_{R}$ is an upper bound on the crack speed [8]. However, in practice, cracks travel slower than $c_{R}$. This has been associated with an instability against branching since the seminal work of Yoffe [9]. She showed analytically that a straight crack is intrinsically unstable at speeds above $\approx 0.66 c_{R}$. The idea is roughly as follows: at low speeds there is stress concentration in front of a crack leading to further motion. However, at high speeds the stress pattern becomes more isotropic, and above a velocity threshold the maximum stresses are at a finite angle to the direction of the tip; this may be assumed to give rise to branching. The effect is due to the fact that the stresses are transmitted by sound waves: if the speed of the crack is too large the crack 'catches up' with the stresses. Other views of the branching instability have been formulated [10] which differ in detail, but not in principle, from Yoffe's work. Simulations of 2-dimensional spring models [6,7] also give a branching instability around $0.66 c_{R}$, and the model we use here has the same property. However, the experiments always show branching at still lower speeds $v_{c} \approx 0.36 c_{R}$ in the case of PMMA, a nearly ideal brittle material; similar observations have been made in ordinary glass [2, 3]. It appears that something is causing branching before the Yoffe mechanism takes hold. Here we introduce a physical effect that has been left out of previous treatments, thermal noise, and show that it could resolve the discrep- ancy.

It is obvious that large enough thermal noise could cause a crack to wander. The essential question is whether reasonable values for the parameters give rise to a substantial effect. A priori this seems unlikely: usually, bonds break due to thermal effects only near the melting point, which is not the situation at hand. However, this is not correct because the nature of the branching is that that even below the threshold the stress pattern near the crack tip is approaching instability, so that a relatively small thermal fluctuation could break the 'wrong' bond, and give rise to branching. We will demonstrate this effect in a simulation where we attempt to fit all of the parameters to experimental values.

To account for thermal noise, we need to have a coupling between the long-wavelength elastic modes, which we describe by a spring model, and a 'heat bath', i.e. the rest of the degrees of freedom of the solid. This arises from a damping or viscosity term. In the macroscopic theory of elasticity damping can be included in the elastic equations of motion by introducing Kelvin viscosity terms. With these terms the equations of motion for the spring model we use [1],12] are:

$$
\begin{aligned}
m \partial_{t t} \mathbf{u}_{\mathbf{r}} & =\sum_{\mathbf{r}^{\prime}} K \hat{\mathbf{n}}\left[\hat{\mathbf{n}} \cdot\left(\mathbf{u}_{\mathbf{r}}-\mathbf{u}_{\mathbf{r}^{\prime}}\right)\right] \\
& +\sum_{\mathbf{r}^{\prime}} \eta \hat{\mathbf{n}}\left[\hat{\mathbf{n}} \cdot\left(\partial_{t} \mathbf{u}_{\mathbf{r}}-\partial_{t} \mathbf{u}_{\mathbf{r}^{\prime}}\right)\right]
\end{aligned}
$$

where $\mathbf{u}_{\mathbf{r}}$ is the displacement of the node at $\mathbf{r}, K$ and $\eta$ are the spring constants and viscosity respectively, $\hat{\mathbf{n}}$ is the unit vector from node $\mathbf{r}$ to $\mathbf{r}^{\prime}$, and the $\mathbf{r}^{\prime}$ 's are the nearest neighbors of $\mathbf{r}$. The viscous terms are the simplest form consistent with the symmetry of the situation [13]. We have previously shown that if $\eta$ is of order unity this term is sufficient to allow a crack to propagate without branching at a terminal velocity less than $v_{c}$. Without viscosity any crack, once initiated in in fixed grip loading conditions (which is the case we consider), accelerates to the branching threshold [11, 12].

The value of $\eta$ is central to our theory. We get it by fitting to the experimental values for the attenuation of longitudinal sound [14]. It is easy to show from Eq. (11) that $\alpha(\omega)$, the inverse of the attentuation length for lon- 
gitudinal sound of frequency $\omega$, is given by $\left(\eta / 2 K c_{L}\right) \omega^{2}$ in our spring model. We define a characteristic frequency, $\omega_{o}=c_{L} / a$ for any elastic system, where $c_{L}$ the speed of longitudinal sound, and $a$ is the length unit, which we take to be the radius of the crack tip. (It is well known that any fracture model must introduce some microscopic length unit.) Then we put $\alpha a=Q\left(\omega / \omega_{o}\right)^{2}$. The dimensionless number $Q$ is a measure of the size of the damping. For the spring model, in units where $K=m=a=1$ we find that $Q=0.53 \eta$. For $\mathrm{SiO}_{2}$ the sound attenuation has also goes like $\omega^{2}$ over a large frequency range [14]. Using the measured data in Ref. [14] for $\alpha$ and $c_{L}$ and using $a \approx 0.5 \mathrm{~nm}$, a molecular dimension, and we find $Q \approx 0.4$. For the case of PMMA the attenuation does not have the same frequency dependence [15], and we cannot unambiguously determine $\eta[11,12]$. In the remainder of this paper we will mostly discuss $\mathrm{SiO}_{2}$. For this case we are justified taking $\eta$ to be of order unity. In all of our simulations we take $\eta=2$.

In order to simulate fracture we take Eq. (11) as the dynamics of a snapping spring model: If any bond is stretched more than $u_{t h}=0.1 a$ we break it. Strips of lattice along the $x$ direction are stressed by holding two edges parallel to the $y$-axis at fixed displacement, $\Delta$, and a crack is started parallel to the $x$-axis. We artificially cut the springs at an effective crack velocity of 0.05 [11], 12 at the center of the width along the length. We solve the equations of motion by using a Verlet algorithm. As soon as velocity exceeds 0.1 the artificial scissoring is stopped. The results are unaffected by this procedure. The width ( $y$-direction) along which the strain is applied is up to $100 a$ and the length ( $x$-direction), along which the crack propagates is up to $400 a$. For more details of our procedure see [11.12].

We first show that the Yoffe instability, or something very much like it, is in fact present in our model. We create cracks at various terminal velocities by varying $\Delta$, and measure the ratio of the strain in the 'right' bond (corresponding to a straight crack) and the 'wrong' one. This is shown in Fig. (1). The ratio goes through unity and the crack branches at $\approx 0.7 c_{R}$.

Now we add thermal noise by putting a random force term, $\theta(\mathbf{r}, t)$, on the right hand side of Eq. (11). The components of the force are independent gaussian noises at each site whose amplitude is chosen to be consistent with the fluctuation-dissipation theorem:

$$
<\theta_{i}(\mathbf{r}, t) \theta_{j}\left(\mathbf{r}^{\prime}, t^{\prime}\right)>=\frac{2 k_{B} T \eta}{d t} \delta_{t, t^{\prime}} \delta_{r, r^{\prime}} \delta_{i, j}
$$

where $i, j$ label the components. The dependence on $1 / d t$ is the standard way to normalize a discrete $\delta$-function.

Strictly speaking the noise should have nearest neighbor correlations in order to be consistent with our form of $\eta$. Put another way, short wavelength modes have more damping than long wavelengths as mentioned above, and we should drive them more. Since bonds are broken by short wavelength fluctuations, we are underestimating the effect of noise by neglecting the correlations. We have done a few runs with the completely consistent noise to verify this. The difference is not very large.

We will need to know the energy unit for our model, $K a^{2}$, in order to evaluate the noise strength. This we get by fitting $K u_{t h}^{2} / 2 a^{2}$ to the fracture energy, $8 \mathrm{~J} / \mathrm{m}^{2}$ for $\mathrm{SiO}_{2}$ [1]. Taken literally, this would give each bond about $20 \mathrm{eV}$, which is too large by a factor of 10 or so. In our units room temperature comes out to be of order $10^{-5}-10^{-6}$. We put $k_{B} T=3 \cdot 10^{-6}$. By taking a large energy unit we are once more underestimating the noise strength.

In the vicinity of the tip there are large local heating effects in some materials including PMMA 16]. To account for this we start the lattice with an ambient temperature (room temperature) and allow it to locally heat (and diffuse heat) from the work of the viscous forces. In that case the temperature becomes a local variable $T(\mathbf{r})$. In order to find $T(\mathbf{r})$ we set:

$$
\partial_{t} T(\mathbf{r})=\frac{1}{C}\left[\left(\mathbf{f}_{v}(\mathbf{r})-\theta(\mathbf{r})\right] \cdot \partial_{t} \mathbf{u}_{\mathbf{r}}+\kappa \nabla^{2} T(\mathbf{r})\right.
$$

Here $\kappa$ is a thermal conductivity, $C$ is the heat capacity associated with the local heat bath, and $\mathbf{f}_{v}$ is the viscous force on node $\mathbf{r}$ (the second term on the right-hand side of Eq. (11). If $C$ is very large the lattice remains at the ambient temperature. In our simulations we adjust $C$ and $\kappa$ so that the vicinity of the tip heats up to about three times room temperature.

The simulation precedure is similar to that in the noisefree case outlined above. We use a version of the Verlet algorithm modified to account for the random forces $\theta_{i}(\mathbf{r}, t)$. An initial crack of length $50 a$ is created and the lattice is allowed to thermalize. After the temperature has settled down, the lattice is strained to the desired value.

Our results are as follows. Since most of the detailed observations in the literature are for PMMA, we will compare to the qualitative features seen in that material, even though the quantitative fitting that we have done is for glass. Fig. (2a) shows the effect of thermal noise on the crack velocity. Noise leads to fluctuations in the crack velocity, an effect that is experimentally observed [5, 3]. The large change in the velocity in the figure marks the change in the row of propagation. As is seen from the figure, there is small rise in the velocity before it saturates. This is also observed when the fracture energy is low [6]. In Fig. (2b) we show two cracks, one without noise and one with, in otherwise identical conditions.

With noise there is a finite probability of wandering for any $v$. Thus we must define the critical velocity in terms of the roughness of the crack. We call $v_{c}$ the velocity above which the roughness is more than $10 \%$ of the width. Beyond this point the fluctuations grow quickly and saturate due to the finite size of the sample. This 
observation is consistent with the experiments [5]. Fig. (3) shows the effect of thermal noise on $v_{c}$. Here the rms deviations are measured from the change of direction and are averaged over 5 runs for each point. The critical velocity for crack wandering is lower than in the absence of noise and is around $0.45 c_{R}$.

The effect of crack wandering from one plane to another causes the rough interface. The experiments 5 . show that at higher velocities the roughness of the cracked surface is larger. Similar effects occur [17] for simulations of crack propagation in silicon. The nature of the wandering instability on our simulations is interesting. Just above $v_{c}$ the crack does not change its direction of propagation. In our simulations this occurs for velocities higher than $0.7 c_{R}$. At lower $v$ the crack wanders by jumping rows to planes adjacent to the initial one. That is, as the velocity increases in the presence of noise, the stress field component $\sigma_{y y}$ fluctuates and before $\sigma_{x x}$ becomes comparable to $\sigma_{y y}$ the crack ceases be confined to the initial plane. In Fig. (位) we plot relative displacements in the $y$ direction for two cases. Fig. (4a) shows these displacements just before the crack begins to propagate. Fig. (बb) is same plot but just before the first wandering is encountered. In the second case the relative displacement in $y$-direction is higher in the row close to the middle one in which crack is propagating. Recent experiments 2 clearly show similar observations. There are two velocity thresholds, $v_{c 1}$ and $v_{c 2}$ such that for velocities $v_{c 1} \leq v \leq v_{c 2}$ surface roughens with almost negligible branching while above $v_{c 2}$ it branches. The value of $v_{c 2}$ is around $0.67 c_{R}$, (the Yoffe velocity) where $\sigma_{x x}$ becomes comparable to $\sigma_{y y}$.

In Fig. (5) we show $v_{c}$ as a function of the heat capacity of the heat bath. When $C$ is large the tip of the crack remains at the ambient temperature. We see that about half of the reduction of $v_{c}$ is due to tip heating for our parameters. In this paper we have used a naive model to treat fracture in $\mathrm{SiO}_{2}$. In fact, Griffith used exactly the same sort of considerations in his pioneering work on glass [1]. We think that the qualitative results of this study will survive a more detailed look at the theory, and that the effect of thermal fluctuations on $v_{c}$ is potentially quite large. The real test for our ideas must come from experiment. For example we could imagine looking at the branching threshold systematically as a function of temperature, or, in materials like PMMA where the polymer chains vary in length, as a function of heat capacity.

We would like to acknowledge useful conversations with O. Pla, F. Guinea, E. Louis, J. Fineberg, M. BenAmar, and M. Adda-Bedia. Supported by NSF grant DMR 94-20335. S. Ghaisas thanks the University of Michigan for hospitality.
[1] B. Lawn Fracture of brittle solids, Cambridge University Press, 1993.

[2] J.F.Boudet and S. Ciliberto, Phys. Rev. Lett. 80, 341 (1998).

[3] E.Sharon, S.P. Gross and J. Fineberg, Phys. Rev. Lett. 76, 2117 (1996).

[4] E.Sharon, S.P. Gross and J. Fineberg, Phys. Rev. Lett. 74, 5096 (1995).

[5] J.F.Boudet, S.Ciliberto and V. Steineberg, Euro Phys. Lett. 30, 337 (1995).

[6] M. Marder and S.P.Gross, J. Mech. Phys. Solids 43, 1 (1995).

[7] F.F.Abraham, Phys. Rev. Lett. 77, 869 (1996).

[8] L.B.Freund, Dynamical Fracture Mechanics (Cambridge Univ. Press, New York ,1990).

[9] E.H.Yoffe, Phil. Mag. 42, 739 (1951).

[10] M. Adda-Bedia, R. Arias, M. Ben-Amar, and F. Lund preprint (1998).

[11] O. Pla, F. Guinea, E.Louis, S.V.Ghaisas and L.M.Sander, Phys. Rev. 57, R13981 (1998).

[12] O. Pla, F. Guinea, E.Louis, S.V.Ghaisas and L.M.Sander, to be published.

[13] L. Landau and E. Lifshitz, Theory of Elasticity, AddisonWesley, 1959.

[14] T. Zhu, H.J.Maris, and J. Tauc Phys. Rev. B44,4281 (1991).

[15] C.J.Morath and H.J.Maris, Phys. Rev. B 54, 203 (1996).

[16] K.N.G.Fuller, P.G.Fox and J.E.Field, Proc. R. Soc. Lond. A 341, 537 (1975).

[17] D.Holland and M.Marder, Phys. Rev. Lett. 80, 746 (1998).

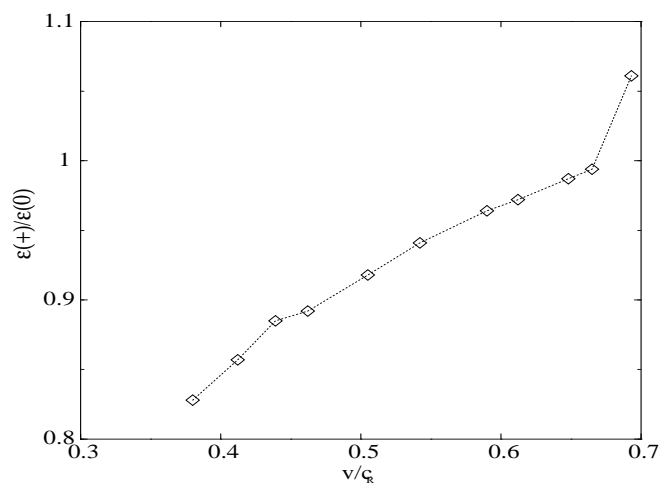

FIG. 1. The branching instability as a function of $v$. The strain in the row corresponding to forward growth is compared to that in the next row. 

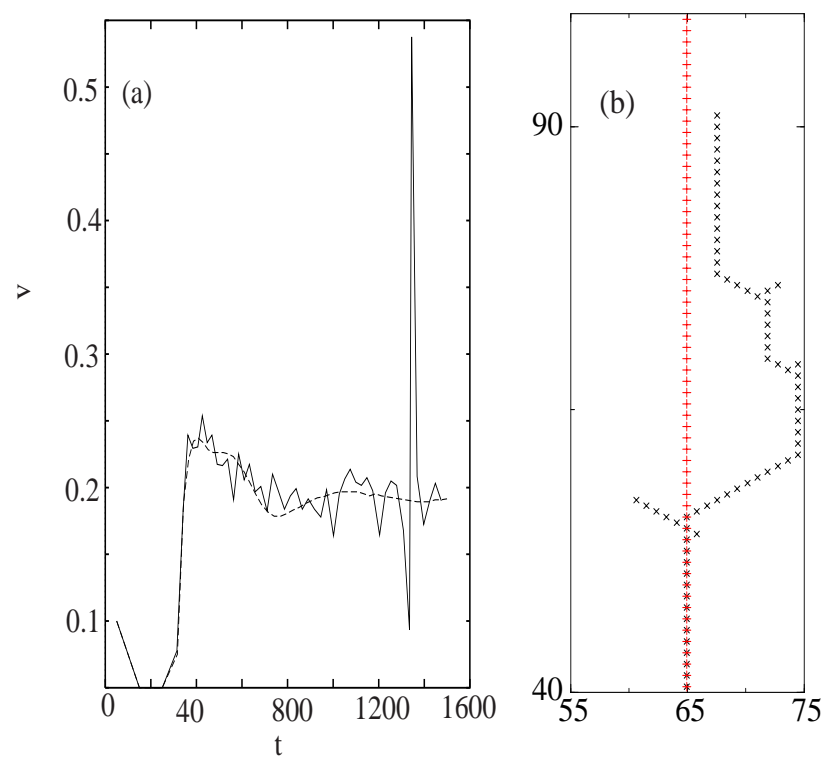

FIG. 2. Effect of noise on crack propagation. (a.) Dashed line is the velocity for a $50 \times 400$ lattice for $\mathrm{T}=0^{0} \mathrm{~K}, \eta=2.0$, specific heat $C=100, \kappa=4.0$, and stretch per unit width $\Delta / W=0.033$ where $W$ is the lattice width. The solid line is the velocity for the same parameters with $T=3.0 \cdot 10^{-6}$ (b.) Crack pattern with and without noise in the same loading conditions

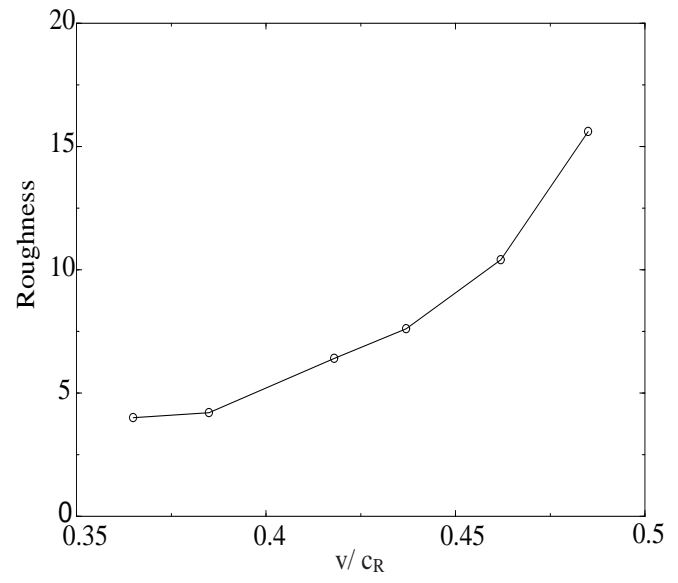

FIG. 3. Rms deviations in the crack path as a function of velocity for $\eta=2.0, C=100, \kappa=4.0, T=3.0 \cdot 10^{-6} . \Delta$ is varied to obtain the appropriate velocity.
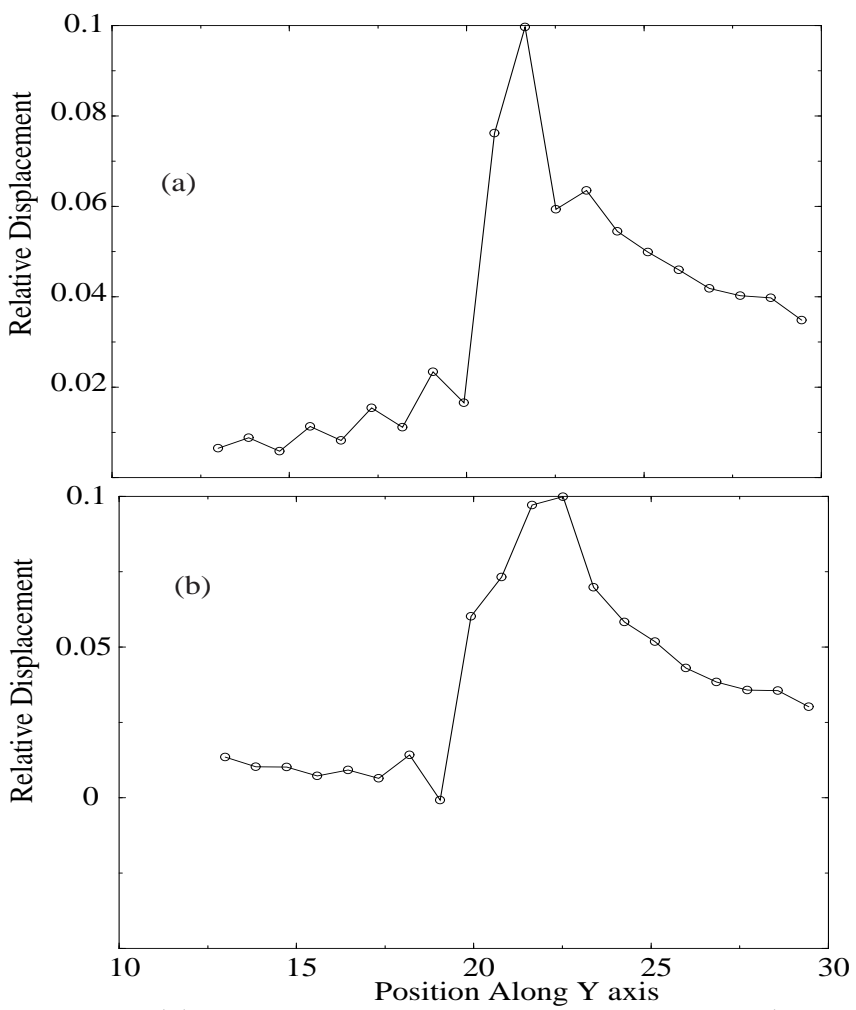

FIG. 4. (a) Relative displacements in the columns (along $y$ direction) containing the crack tip and one column ahead just before the crack begins to propagate. (b) Relative displacements in the columns containing the tip just before first wandering is encountered. The parameters are the same as in the previous figures.

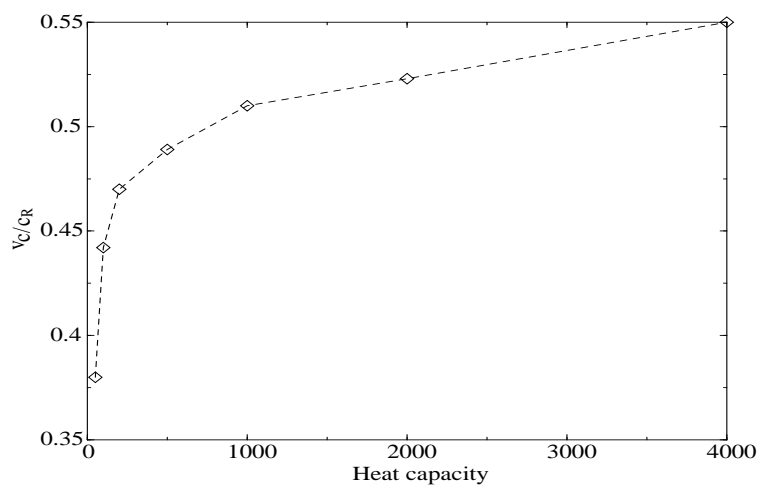

FIG. 5. Critical velocity as a function of heat capacity. For large heat capacity local heating in the vicinity of the tip is suppressed. 